\title{
Bluetooth-Based Sensor Networks for Remotely Monitoring the Physiological Signals of a Patient
}

\author{
Ying Zhang and Hannan Xiao
}

\begin{abstract}
Integrating intelligent medical microsensors into a wireless communication network makes it possible to remotely collect physiological signals of a patient, release the patient from being tethered to monitoring medical instrumentations, and facilitate the patient's early hospital discharge. This can further improve life quality by providing continuous observation without the need of disrupting the patient's normal life, thus reducing the risk of infection significantly, and decreasing the cost of the hospital and the patient. This paper discusses the implementation issues, and describes the overall system architecture of our developed Bluetooth sensor network for patient monitoring and the corresponding heart activity sensors. It also presents our approach to developing the intelligent physiological sensor nodes involving integration of Bluetooth radio technology, hardware and software organization, and our solutions for onboard signal processing.
\end{abstract}

Index Terms-Bluetooth, healthcare, physiological signals, wireless sensor network.

\section{INTRODUCTION}

I $\mathrm{N}$ RECENT years, the focus of health policy has been shifting from providing more reactive and acute healthcare in hospital toward providing more proactive and preventive healthcare outside hospital [1], [2]. Because emergency admission to hospital and lengthy stay at hospital are extremely costly, and do challenge the available medical resources, the mode of healthcare is being innovated to reduce and avoid these circumstances. In fact, the reason for staying in hospital, in most cases, is not because a patient actually needs active medical treatment, but often because the patient needs continuous observation for reliably detecting the progressive abnormalities (e.g., the symptoms of a chronic disease), timely monitoring the therapy effects, or avoiding occurrence of adverse events in the recovery process of postsurgery. Meanwhile, early detection of health anomalies can significantly reduce the emergency admissions to large acute centers.

Therefore, medical society is seeking the technologies to be able to advance healthcare by early detection of health anomalies and by early discharge of patients from hospital with continuous out-hospital observation. This has potential for not only reducing the risk of cross-infection, but also significantly decreasing the cost of hospitals. However, most of current medical systems

Manuscript received October 18, 2008; revised March 18, 2009. First published September 1, 2009; current version published November 4, 2009. This work was supported by a generous grant from the Modernization Initiatives with the support of the Guy's and St Thomas Charity.

Y. Zhang is with the Engineering Department, University of Cambridge, Cambridge CB3 0FA, U.K. (e-mail: yz282@ cam.ac.uk).

H. Xiao is with the School of Computer Science, University of Hertfordshire, Hatfield, Hertfordshire AL10 0HY, U.K. (e-mail: h.xiao@ herts.ac.uk).

Color versions of one or more of the figures in this paper are available online at http://ieeexplore.ieee.org.

Digital Object Identifier 10.1109/TITB.2009.2028883

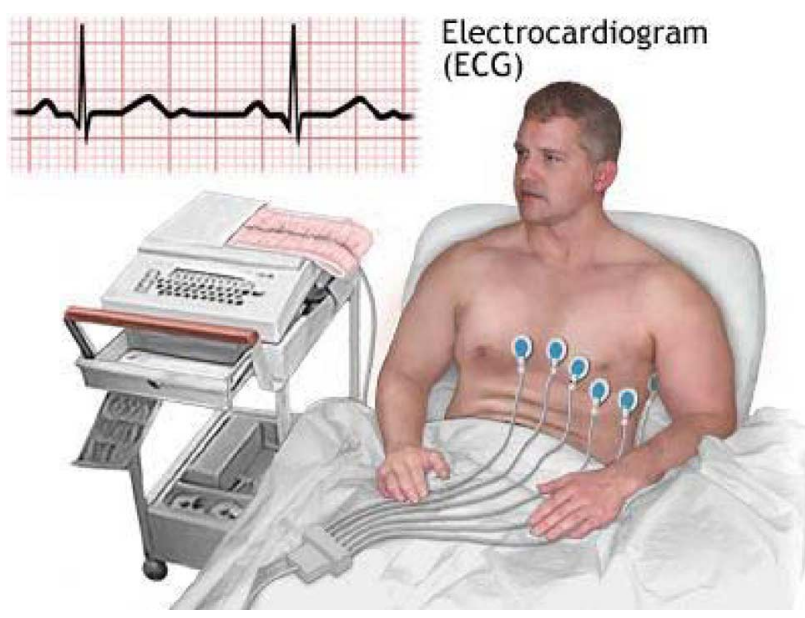

Fig. 1. Traditional physiological signal measurement.

and instrumentations need tether the patients by unwieldy wires connected to a confined area in the hospital so that the patients' physiological signals can be measured and monitored for diagnosing a disease or for assessing the treatment effects (see Fig. 1). These factors may limit the patients' activity, decreasing the level of comfort, and thus, negatively affecting measure and diagnostic effects.

Wireless sensor network technology provides a useful method to remotely acquire and monitor the physiological signals without the need of disrupting the patient's normal life, thus improving life quality [3]-[8]. Furthermore, integrating diverse medical sensors together can combine medical information from multiple sources, thus improving diagnostic accuracy of a disease, simplifying diagnostic procedure, and discovering potential knowledge through data fusion of all gathered information. Actually, recent technology advances in wireless communication, micropower medical sensors, and network technologies make it possible to design the new-generation healthcare system, which brings together the abundance of existing specialized medical technology with pervasive, wireless networks, and lowcost, miniature, lightweight, intelligent physiological sensors.

However, most of recent efforts in health systems confine to either physiological signal acquisition, data processing, and interpretation [9]-[16], or the advance of a special aspect such as networking challenges [17], medical sensors [18]-[20], physiological data management system [21], and sensor node design. For instance, BTnode [22]-[26] from Eidgenössische Technische Hochschule Zurich is a prototyping platform for ad hoc networks. It consists of an Atmel ATmega128 microcontroller and two separate radio modules. One is a low-power Chipcon CC1000-based [27] industrial, scientific, and medical (ISM)band broadcast radio module, which operates in the frequency 
range of 433-915 $\mathrm{MHz}$ and uses proprietary protocols. The other is a Zeevo ZV4002-based [28] Bluetooth radio module. It supports Bluetooth specification version 1.2. The Zeevo Bluetooth radio module can be operated using parts of the Bluetooth protocol stack. Its Bluetooth stack supports the host controller interface (HCI), Logical Link Control and Adaptation Protocol (L2CAP), and RF communication (RFCOMM) layers, thus allowing for some interoperability with other Bluetooth devices. BTnode uses a threaded Nut/OS [29] operating system for scheduling multiple threads, basic memory management, events, synchronization, and streaming I/O and device drivers. However, it does not support Bluetooth Service Discovery Protocol (SDP). This requires users to manually configure all connections, and thus, places the BTnode in between standardsbased and proprietary architectures.

Telos [30], [31] is the new generation of wireless sensor device (i.e., mote) from the University of California (UC) Berkeley. It features low power consumption, easy-to-use, and robustness. It uses Texas Instruments (TI) Incorporated's MSP430 microcontroller and Chipcon CC2420 [32] radio transceiver. MSP430F1611 [33] is a 16-bit reduced instruction set computer (RISC) processor with a built-in eight-channel 12-bit A/D converter, serial peripheral interface (SPI) and universal asynchronous receiver/transmitter (UART) peripherals, a twochannel 12-bit D/A converter, a supply voltage supervisor, and a direct memory access (DMA) controller. It can be operated at 6 $\mathrm{MHz}$, and has 10000 bytes on-chip RAM, 48000 bytes built-in flash memory, and 128 bytes of information storage. Chipcon CC2420 is an IEEE 802.15.4 compliant radio transceiver that operates in $2.4 \mathrm{GHz}$ frequency band, and provides the physical layer (PHY) and some media access control (MAC) functions. The CC2420 is controlled by the TI MSP430 microcontroller through the SPI port and a series of digital I/O lines and interrupts. The radio module can be shut off by the microcontroller for low-power duty-cycle operation. Telos mote uses TinyOS [34] operating system.

The iMote2 was developed by Intel and UC Los Angeles. It is based on PXA271 Xscale processor and uses a Chipcon CC2420 radio transceiver, which is a Zigbee (i.e., IEEE 802.15.4) radio transceiver. This platform is stackable and allows for a multitude of different sensors and power supplies to be attached. It has $32 \mathrm{MB}$ onboard flash memory and $32 \mathrm{MB}$ onboard static RAM (SRAM). iMote uses TinyOS operating system. It does not support standard Bluetooth profiles, but adopts the customized protocol layers that are written for TinyOS. These layers provide support for topology establishment and formation of both single- and multihop networks. The use of TinyOS simplifies code reuse from other non-Bluetooth mote platform. More information about iMote2 can be found in [35].

Milenkovic et al. [4], [5] presented the architecture, the issues, and the implementation of a prototype of a wireless sensor network for personal health monitoring; Chan et al. [36] implemented a wireless sensor network for assessing the care needs of residents in aged-facilities with multiple sensors: ECG, triaccelerometer, body temperature, and light intensity. Gondal et al. [37] proposed a sensor network architecture that integrates sensing and diagnosis methods together. Spadini et al. [38] implemented a portable monitoring system for ECG data; this system also integrates the subject's activity from accelerometers and environmental data like temperature, humidity, and light intensity, giving a better general picture of where the changes of ECG data come from. However, these systems have the common disadvantage of wearability and lack of the combination with medical data management systems.

The wireless health system described in this paper has several key novelties: wearability, reconfigurability, and integration with medical data management system. It brings together traditional medical technology with recent advances in wireless communication, micropower medical sensors, medical data management, and network technologies. This system captures physiological signals from a patient who requires constant monitoring, and then, transmits the collected data to a medical center or a hospital where medical personnel can remotely observe the patient's health status. National Semiconductor's CP3SP33 connectivity processor is adopted to design the sensor node and the coordinator node. The main reasons for choosing CP3SP33 are the following: 1) it is a 16-bit processor; 2) it has an on-chip Bluetooth peripheral, a Universal Serial Bus (USB) 2.0 peripheral, and a 10-bit A/D converter; and 3) it supports different low-power modes to reduce power consumption in different operating situations.

This paper describes the overall system architecture of the developed sensor network and the corresponding heart activity sensors. It presents our approach to developing the intelligent physiological sensor nodes, and our methods to integrate Bluetooth technology into these sensor nodes, hardware and software organization, and our solutions for onboard signal processing. This paper also discusses the implementation issues.

The remainder of this paper is organized as follows. Section II presents the overall system architecture. Section III describes the wireless personal area sensor network, the medical sensor node, and the coordinator node, respectively. Section IV presents the relevant software organization. Section $\mathrm{V}$ describes the application scenarios and initial evaluation. Finally, the conclusions are given in Section VI.

\section{OverAll SYSTEM ARCHITECTURE}

This wireless medical system consists of three major parts: medical sensors (i.e., sensor nodes), gateway module, and medical server and the medical personnel's terminals at remote location (i.e., hospital). The diagram of the whole system is shown in Fig. 2.

The part of medical sensors includes several sensor nodes. Each sensor node may have several medical sensors. It is equipped with the physiological signal conditioning circuits, a microcontroller, and a Bluetooth interface. Each sensor node can be configured to sense, sample, and process one or more physiological signals. For example, an ECG sensor can be used to collect the physiological signals for monitoring heart activity, an EEG sensor for monitoring brain electrical activity, an electromyography (EMG) sensor for monitoring muscle activity, a blood pressure sensor for monitoring blood pressure, a blood glucose sensor for measuring the density of glucose, a 


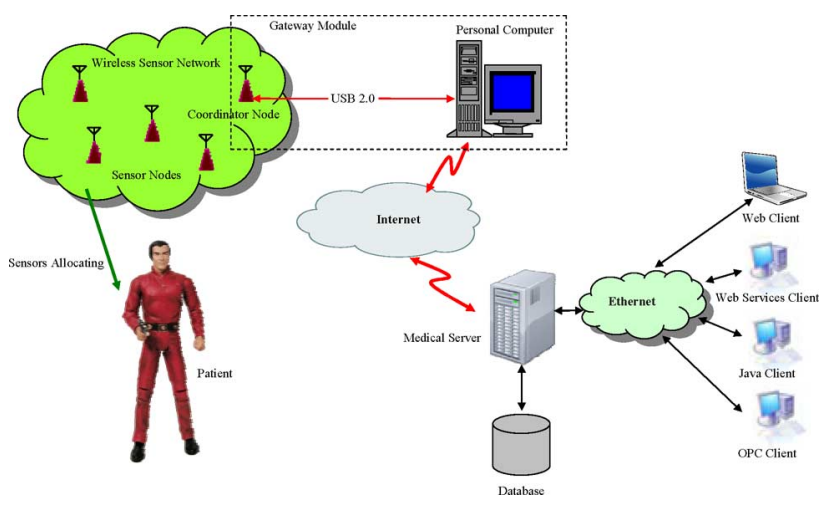

Fig. 2. Overall system architecture.

temperature sensor for monitoring the body temperature, a tilt sensor for monitoring trunk position, and a breathing sensor for monitoring respiration. Motion sensors can be used to discriminate the patient's status and estimate the level of activity. In addition, some nonmedical sensors can be integrated into this system architecture to acquire the environmental data like temperature, humidity, and light intensity. These data may give a better understanding where the patient is and whether these factors affect the physiological data.

These sensors can be selectively configured to monitor the respective physiological signal depending on the diagnostic demands of a patient's disease. The patient's physiological signals are collected by the medical sensors, initially processed by these sensor nodes at the patient end, and then, the preprocessed data are transmitted to the coordinator node, which is a part of gateway module, via Bluetooth connection.

The gateway module consists of two major parts: a coordinator node with Bluetooth and USB interface, and a home computer with USB and Internet interface (or a cell phone/a personal digital assistant (PDA) with USB and general packet radio service (GPRS) [39], [40] access interface). The coordinator node and medical sensor nodes form a Bluetooth piconet that includes up to seven slave medical sensor nodes and a master node.

Here, the coordinator node always acts as a master. If the patient has a home computer that is configured as a part of gateway module, the gateway module communicates with medical sensor nodes via Bluetooth connection, and communicates with the medical server through the Internet (see Fig. 2). If the patient has a PDA or a cell phone that is configured as a part of gateway module, the gateway module communicates with medical sensor nodes via Bluetooth connection as mentioned before, but communicates with the medical server through GPRS (see Fig. 3). Within the gateway module, the coordinator node communicates with the patient's home computer, PDA, or cell phone through the USB interface. Sequentially, the data are communicated from gateway module to the medical server through the Internet, wireless local area network (WLAN), or GPRS. The choice would largely depend on whether the patient has a computer. The medical data are stored in medical sever and can be accessed by medical personnel from their computer terminals that are connected to the medical server via local Ethernet.

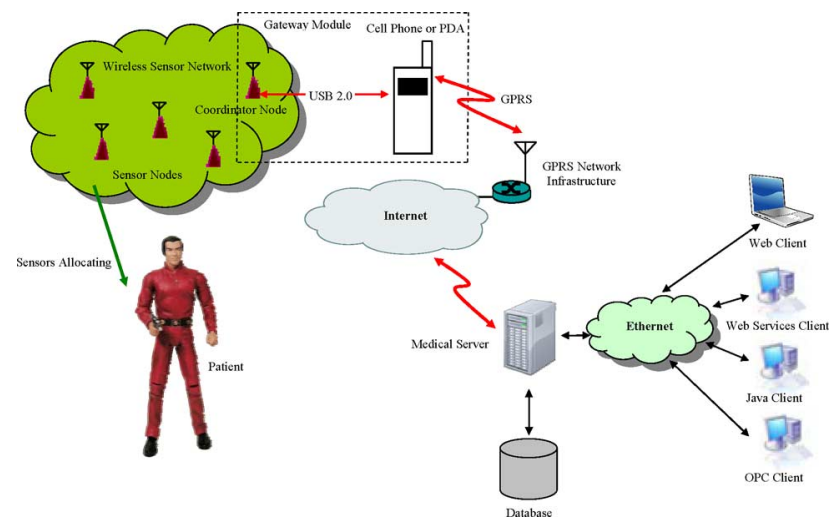

Fig. 3. System configuration with GPRS.

This paper describes our concept-proof prototype system. The system architecture is shown in Fig. 2. It only monitors ECG signals and needs the patient to have a home computer to be a part of gateway module. More physiological sensors can be easily further integrated into this system framework, and more data transmission methods can be adopted, such as GPRS.

In the medical server and medical personnel's computer terminals, a Web-based ECG management system, which offers the solutions for managing ECGs and testing this prototype system architecture, is developed. Open-source freeware with good performance and stability, such as Apache, Hypertext Preprocessor (PHP), and My Structured Query Language (MySQL), was used to expedite the development and minimize the cost. In order to test the prototype system, a simple ECG file structure and GUI are designed to store and visualize the ECG data on the patient's home computer. After the entire system architecture is verified, the next step would be to integrate some medical signal analysis modules, such as ECG pattern recognition, and the modules for statistical/history analysis into this system. Then, more modules for other physiological data such as blood pressure and EEG will be brought in. Hope this system can finally merge with and be a part of the medical data management system.

\section{WIRELESS MEDICAL SENSOR NETWORK}

\section{A. Medical Sensor Network}

Traditionally, a couple of wires are used to connect diverse sensors to a portable/wearable medical device for monitoring and collecting physiological signals. All these medical sensors form a wired personal area network to facilitate the integration of medical information from multiple sources. However, this architecture is unsuitable for longer and continuous monitoring, particularly when patients hope to maintain normal activities without disrupting their daily lives. The system described in this paper uses a couple of medical sensors to build a wireless personal area network and collect multiple physiological signals.

This wireless personal area sensor network is a Bluetooth piconet. Bluetooth is a low-cost, short-range wireless technology with small footprint, low power consumption, reasonable throughput, and hence, suitable for various small, battery-driven 


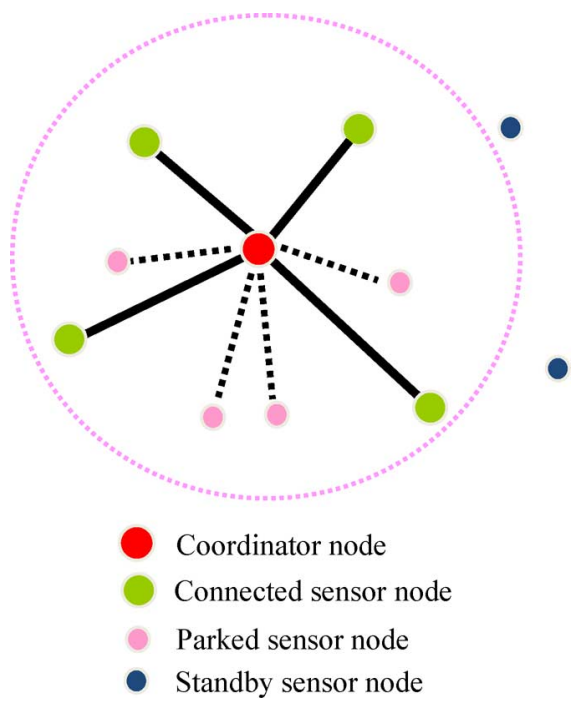

Fig. 4. Piconet formed by a coordinator node and several sensor nodes.

devices. Bluetooth operates in $2.4 \mathrm{GHz}$ frequency band and uses frequency hopping spread spectrum (FHSS) technique. There are 79 channels, each $1 \mathrm{MHz}$ wide, available for hopping [41]. It supports frequency hopping up to $1600 \mathrm{hops} / \mathrm{s}$. ZigbBee protocol is similar to Bluetooth in that both run in the $2.4 \mathrm{GHz}$ unlicensed frequency band and both use low power. However, ZigBee aims more for large-scale automation and remote control, while Bluetooth focuses more on user mobility and eliminating short-distance cabling. In addition, Zigbee's maximum data rate is 250 only $\mathrm{kb} / \mathrm{s}$, which could be a potential problem for the data collection in the proposed healthcare network.

This Bluetooth piconet consists of up to seven slave sensor nodes and a coordinator node (i.e., the master node). Each slave sensor node senses, samples, and processes one or more physiological signals, and then, sends the data to the coordinator node. In the Bluetooth piconet, the slave nodes do not communicate with each other directly-all communication must be through the master node. This makes Bluetooth piconet particularly suitable for the medical sensor network where there is normally no communication between sensor nodes, and the gateway needs to collect all the sensor data.

Fig. 4 shows the formed piconet. The coordinator node is prestalled at a fixed location beforehand; it periodically sends out inquiries over the 32 Bluetooth wakeup carriers to see whether nearby sensor nodes want to join this piconet. The inquiry packets contain the inquiry access code (IAC) of a Bluetooth network, which is known to all of the sensor nodes. When a sensor node receives an inquiry from the coordinator node, the sensor node sends back a frequency hopping serial (FHS) packet containing its device address and timing information to the coordinator node. The coordinator node then sends a paging packet with the sensor node's data access code (DAC) back to the paging carriers. After the sensor node receives paging packet, it sends an acknowledgement to the coordinator node, and then, the coordinator node issues its FHS packet to the sensor node. Finally, the sensor node acknowledges the receipt of the coordinator node's FHS packet and the connection is established.

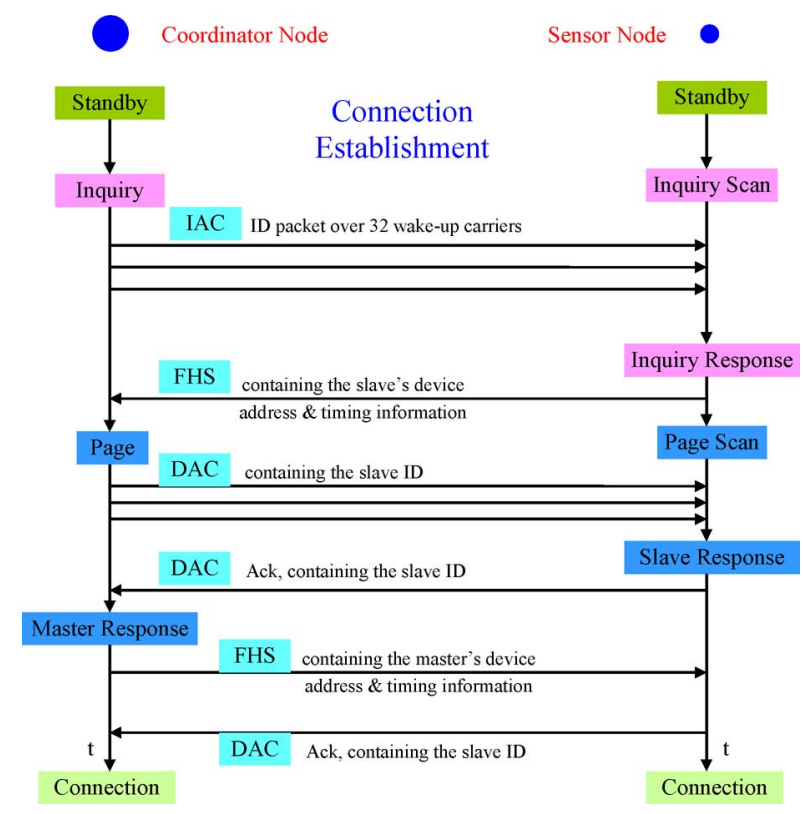

Fig. 5. Connection establishment between the coordinator node and a sensor node.

Fig. 5 shows the connection establishment procedure between the coordinator node and a sensor node, including the exchanged messages and the state transition. In this way, up to seven sensor nodes can join the piconet that is mastered by the coordinator node. Once the piconet is established, the coordinator node polls each sensor node in a round-robin fashion to interrogate whether a sensor node is ready to send the data that are periodically collected and formatted. The coordinator node polls at even timeslots, while the sensor nodes send back at odd timeslots with packets occupying 1,3 , or 5 timeslots; each timeslot is $625 \mu$ s long in Bluetooth FHSS.

If a patient stays in a hospital, medical personnel normally authenticate the patient by asking private information such as name, birth date, and home address, including postcode, before taking any medical treatment. While a patient is remotely monitored, authentication needs to be done via the Bluetooth sensor network. The Bluetooth security defines pairing procedures of three or four steps: creation of an initialization key, creation of a link key, authentication, and derivation of an encryption key [42], [43].

During the first step of creating an initialization key, a personal identification number (PIN) may be generated based on the patient's private information. Before a connection is established, the patient needs to input some private information into the Bluetooth device. A coordinator node should have such information beforehand of any possible sensor nodes. Such authentication method is user-friendly as it does not require the patient to remember a secret number. It also prevents a sensor node from being accidentally played by a child. It is argued here that the encryption of communications between a coordinator node and a sensor node is not so necessary unless the patient is a very important person and the health monitoring is really sensitive data. 


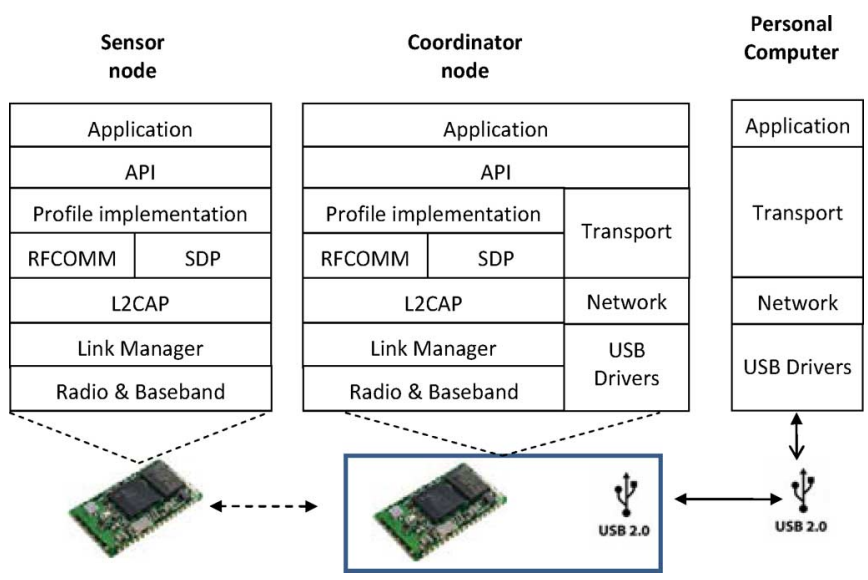

Fig. 6. Bluetooth and USB 2.0 protocol stacks of the coordinator node and the sensor nodes.

Fig. 6 shows the Bluetooth and USB protocol stacks of the coordinator node and the sensor nodes for the corresponding communication between a sensor node and the coordinator node, and inside the gateway module between the coordinator node and the personal computer.

\section{B. Medical Sensor Node}

The medical sensor nodes are responsible for collecting physiological signals from patients by using diverse sensors, and amplifying and digitizing these signals. The data are then preprocessed, formatted, and transmitted to the coordinator node and gateway module at the patient's home before being relayed to the medical server at remote location (e.g., the hospital). Medical sensor nodes work as slave nodes in the wireless personal area network. A medical sensor node comprises three functional blocks: sensing, data processing, and communication. Each sensor node can have several sensors and the respective physiological signal conditioning modules, a microcontroller, and a Bluetooth module. Fig. 7 is the diagram of a medical sensor node.

A National Semiconductor Corporation's CP3SP33 processor [44], which is a typical chip of CP3000 family connectivity processors, is used to implement the medical sensor node. It is a 16-bit processor with a built-in Bluetooth peripheral, a USB 2.0 peripheral, and a ten-channel, 10-bit A/D converter. It can be operated at $96 \mathrm{MHz}$ with zero wait states. It has 4000 bytes CPU instruction cache, 32000 bytes CPU data RAM, 4000 bytes CPU/DSP shared RAM, 24000 bytes DSP program RAM, 24000 bytes DSP data RAM, 8000 bytes Bluetooth sequencer and data RAM, and addresses up to $96 \mathrm{MB}$ of external memory. As shown in Fig. 7, the sensors are directly connected to the respective signal conditioning modules. The output of the conditioning module is then digitized by the 10-bit on-chip $\mathrm{A} / \mathrm{D}$ converter of the CP3SP33 processor, with $10 \mu$ s conversion time. The processor preprocesses the digitized data, and then, transmits the data to the coordinator node through wireless linkage. The linkage is made up of on-chip Bluetooth Lower Link Controller of the CP3SP33 processor, a National Semiconductor

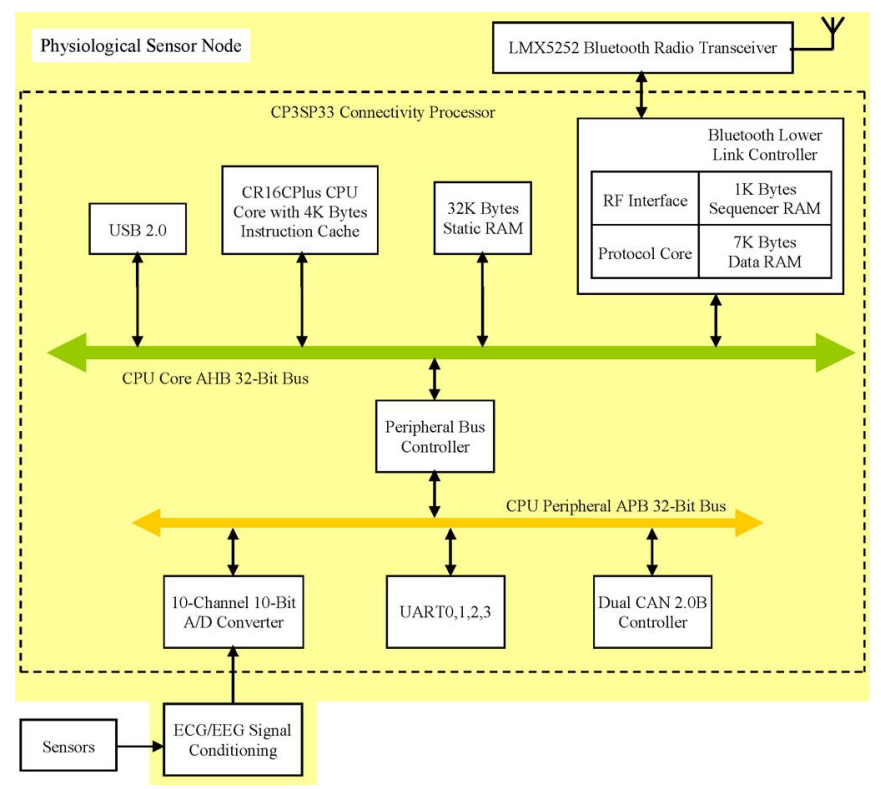

Fig. 7. Diagram of a medical sensor node.

Corporation's LMX5252 Bluetooth radio transceiver [45], and a Yageo's Phycomp-branded $2.4 \mathrm{GHz}$ antenna.

The corresponding firmware is developed for data collection from sensors, management of the Bluetooth peripheral, and communication with the coordinator node. A medical sensor node is reconfigurable depending on the patient's medical condition, and then, these selected sensors are allocated to appropriate parts of the patient's body. For instance, a patient being monitored for a heart condition would have ECG sensors attached to the chest, and a patient being monitored for stroke symptoms might have EEG sensors attached to the scalp and/or EMG sensors attached to various muscles in the body.

This system uses a standard ECG front-end design that can be found in [46]. The sensor node is all integrated on one board. It has several operating modes. The power supply $V_{\text {cc }}=5.0 \mathrm{~V}, V_{\mathrm{I} / \text { Oport }}=3.3 \mathrm{~V}$, and $V_{\text {core }}=1.8 \mathrm{~V}$. The overall current consumption is different in various operating modes: $I_{\text {continous_transmit }}=96 \mathrm{~mA} ; I_{\text {continuous_receive }}$ $=96 \mathrm{~mA} ; I_{\text {receive_data_in } \_ \text {SPP_link }}=32 \mathrm{~mA} ; I_{\text {sniff } \_ \text {mode }}=$ $8.5 \mathrm{~mA} ; I_{\text {scanning_(no_active_link,TL_disabled) }}=0.8 \mathrm{~mA}$; and $I_{\text {idle_(scanning_disabled,TL_disabled) }}=120 \mu \mathrm{A}$. It adopts different low-power modes to reduce power consumption in different operating situations. The modular structure of the node and CP3SP33 microprocessor allow the firmware to power down unused modules. The low-power consumption modes are shown in Table I. The transition between different low-power consumption modes is shown in Fig. 8. The detailed electrical parameters of this sensor node will be reported in another paper under preparation.

\section{Coordinator Node}

The coordinator node is a part of gateway module (see Figs. 2 and 3), which mainly has following functions. 
TABLE I

POWER CONSUMPTION MODE ACTIVITY

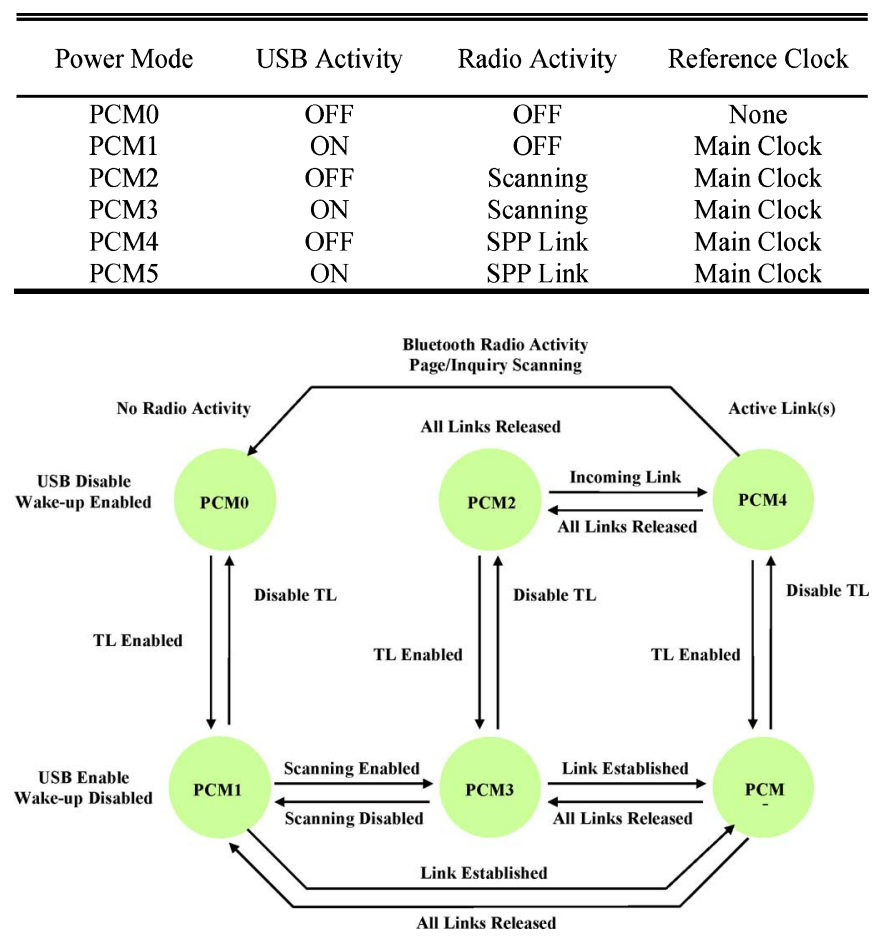

Fig. 8. Transition between different low-power modes.

1) It communicates with the medical sensor nodes, which are attached to the patient, via the built Bluetooth wireless sensor network. The coordinator node actively discovers other medical sensor nodes, and coordinates data transmission to and from sensors.

2) It keeps the captured medical data for self-record. Before being transmitted to the medical server at hospital or medical center, the medical data can be saved in the home computer for self-record.

3) It communicates with the medical server over the Internet, wide-area networks, or some short-range communication technology. When a secure channel to medical server is established, the coordinator node periodically updates the patient's medical record at the medical server.

The coordinator node is implemented by using CP3SP33 connectivity processor. The diagram of the coordinator node is shown in Fig. 9. It has an on-chip USB peripheral and supports USB specification 2.0 to communicate with home computer within the gateway module. A LMX5252 Bluetooth radio transceiver is adopted in the coordinator node. It connects to a Yageo's Phycomp-branded $2.4 \mathrm{GHz}$ antenna.

\section{SOFTWARE ORGANIZATION}

The medical sensor nodes and the coordinator node run an event-driven real-time operating system (RTOS) called $\mu \mathrm{C} / \mathrm{OS}-\mathrm{II}$ [47], which is a portable, ROMable, scalable, multitasking, fully preemptive real-time kernel, and can manage up to 64 tasks. It specifically addresses the concurrency and resource management, and can support the nested interrupts up to

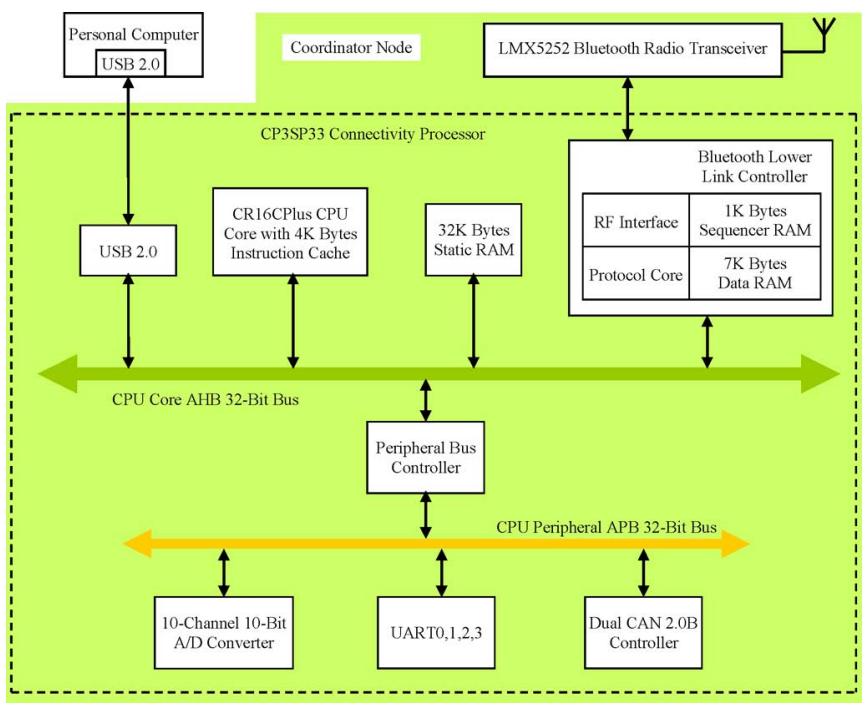

Fig. 9. Diagram of the coordinator node.

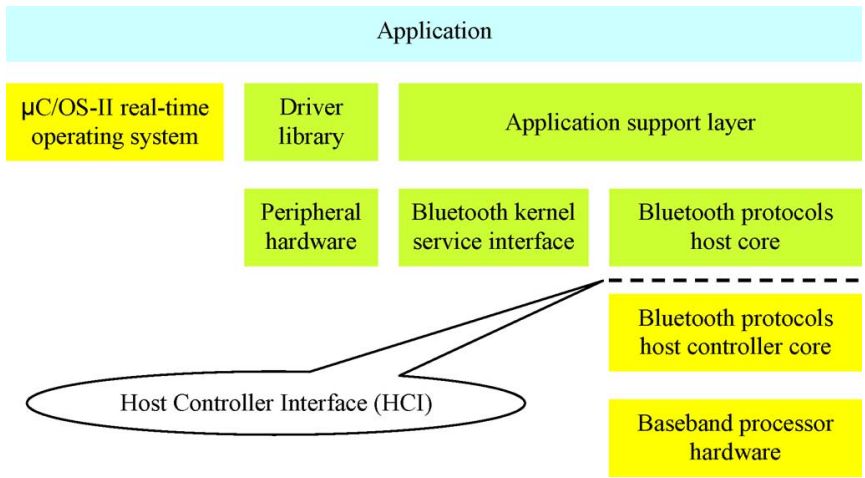

Fig. 10. Software of the sensor nodes.

255 levels deep. The execution time for most services provided by the $\mu \mathrm{C} / \mathrm{OS}$-II operating system is both constant and deterministic. This means that the execution time does not depend on the number of tasks running in the application. The applications such as data processing and Bluetooth communication are written in ANSI C, running on the top of this kernel.

The software of a sensor node consists of three layers, as shown in Fig. 10.

1) RTOS: The sensor node uses $\mu$ C/OS-II RTOS. It provides a multitasking environment with support for taskscheduling, intertask communication, and other services that simplify the design of applications.

2) Driver library: A library of peripheral drivers is developed for interface to the on-chip peripheral hardware of the CP3SP33 connectivity processor and the off-chip peripherals developed by sensor-node-level designs.

3) Application support layer (ASL) (see Fig. 11): This layer is a high-level interface to the Bluetooth kernel and the Bluetooth protocol stack. The Bluetooth kernel is the engine that maintains connectivity by handling activity on network side of the interface, such as scanning for new devices and responding to scans from other devices. The 


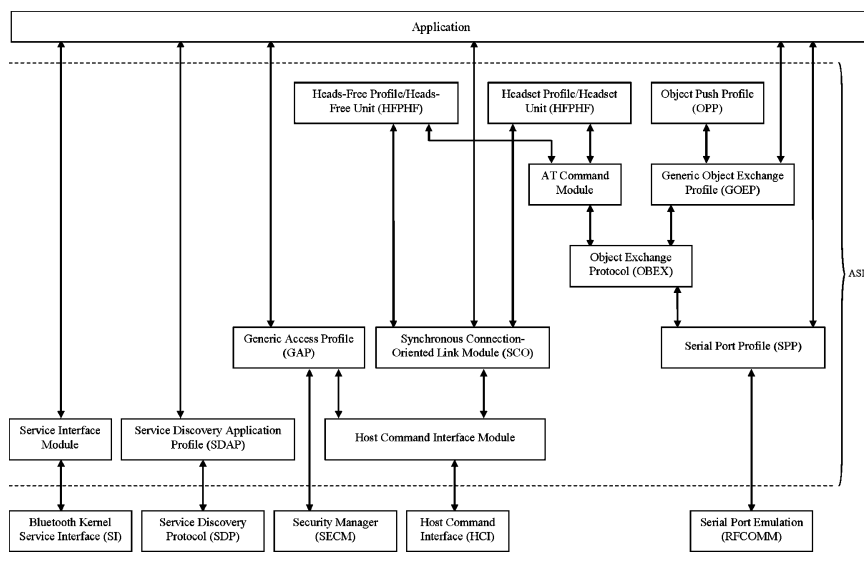

Fig. 11. ASL [48].

interface to the kernel is called service interface (SI). The Bluetooth protocol stack handles application requests for services, such as making connections and transferring data. The upper layers of the stack (above the HCI) are called host core (HC).

These protocols deal with implementing services such as serial port emulation, service discovery, etc., on top of the packetbased communications provided by the HCI, similar to the way that Internet protocols such as Transmission Control Protocol (TCP) and User Datagram Protocol (UDP) implement services on top of IP. The layers below HCI are called host controller core (HCC). These layers implement basic packet communication services for the HCI, and they control Bluetooth hardware (i.e., the baseband processor). The Bluetooth kernel, HC, and HCC are collectively referred to as Bluetooth core.

Although a Bluetooth application could be implemented by making calls directly to the functions that implement Bluetooth protocols, it would be a very low level interface. The sensor nodes in this system use the Bluetooth core provided by National Semiconductor Corporation. This Bluetooth core has a highlevel interface that greatly simplifies the task of implementing a Bluetooth application, called ASL (see Fig. 11). This layer provides all of the functionality that the applications need, but it does not require expert knowledge of the hundreds of function calls and data structures used internally within the Bluetooth core.

\section{A. Bluetooth Protocol Stack}

The baseband and lower link control functions are implemented using a combination of National Semiconductor Corporation's CompactRISC 32-bit processor and the Bluetooth Lower Link Controller. These processors operate from integrated ROM memory and RAM, and execute onboard firmware, implementing all Bluetooth functions and data gathering functions. The integrated Bluetooth Lower Link Controller complies with the Bluetooth specification version 1.2, implementing the functions such as adaptive frequency hopping; interlaced scanning; fast connect; support for 1, 3, and 5 slot packet types; fast frequency hopping at $1600 \mathrm{hops} / \mathrm{s}$; access code correlation; and slot timing recovery.

\begin{tabular}{|l|l|}
\hline \multicolumn{2}{|c|}{ Command Interpreter } \\
\hline \multicolumn{2}{|c|}{ Control Application } \\
\hline SPP & \multicolumn{2}{|c|}{ SDAP } \\
\hline \multicolumn{2}{|c|}{ GAP } \\
\hline RFComm & SDP \\
\hline \multicolumn{2}{|c|}{ L2CAP } \\
\hline \multicolumn{2}{|c|}{ Link Manager } \\
\hline \multicolumn{2}{|c|}{ Radio \& Baseband } \\
\hline
\end{tabular}

Fig. 12. Bluetooth stack protocol.

The integrated upper layer stack is prequalified and involves three protocol layers: L2CAP, RFCOMM, and SDP. It supports Generic Access Profile (GAP), Service Discovery Application Profile (SDAP), and Serial Port Profile (SPP). Fig. 12 shows the Bluetooth protocol stack with command interpreter interface. The command interpreter offers a number of different commands to support the functionality given by the different profiles. Execution and interface timing is handled by the control application. The firmware resides inside the CP3SP33 connectivity processor. The corresponding application programming interface (API) can be referenced in [48].

\section{B. Communication With Medical Sensors}

On the top of the Bluetooth link, a simple, but very flexible data exchange protocol, XMLSense (Extensible Markup Language (XML)-based protocol for data exchange with sensors) [49], is used for transmitting and receiving data to and from sensors.

Using two types of messages, the gateway module can request either the list of sensors attached to a particular medical sensor node or the captured physiological data. Reply messages have very flexible structure and can relay information about any number or type of sensors. Sensor node profiles are defined according to an XML scheme, and are sent as XML strings in reply messages. As all relevant information about a sensor node is contained in its profile (sensor type, measuring unit, accuracy, manufacturer, calibration date, etc.), gateway module can automatically build knowledge of the sensor network and its characteristics, that is to say, sensor nodes can be deployed in an ad hoc fashion.

Bluetooth links are maintained as long as the gateway module and the medical sensor node are in the range. This approach has its advantages and disadvantages. Good side is that as soon as an event happens on a sensor node, the sensor node can send information to the gateway module about the event. Drawbacks are that power resources are wasted on maintenance of communication link, and it is not possible to have more than seven sensor nodes in one piconet.

\section{APPLICATION SCENARIOS AND INITIAL EVALUATION}

There are two typical deployment scenarios for this system. It can be deployed at home or in a hospital. For the deployment 


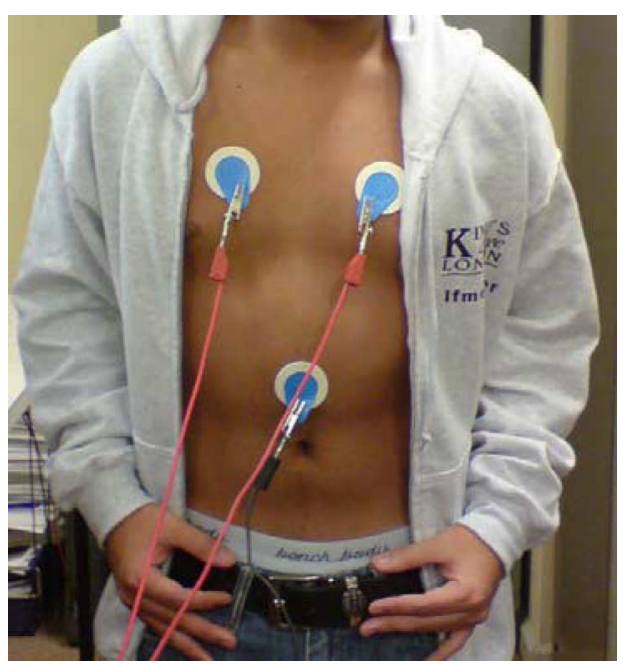

Fig. 13. ECG signal monitoring.

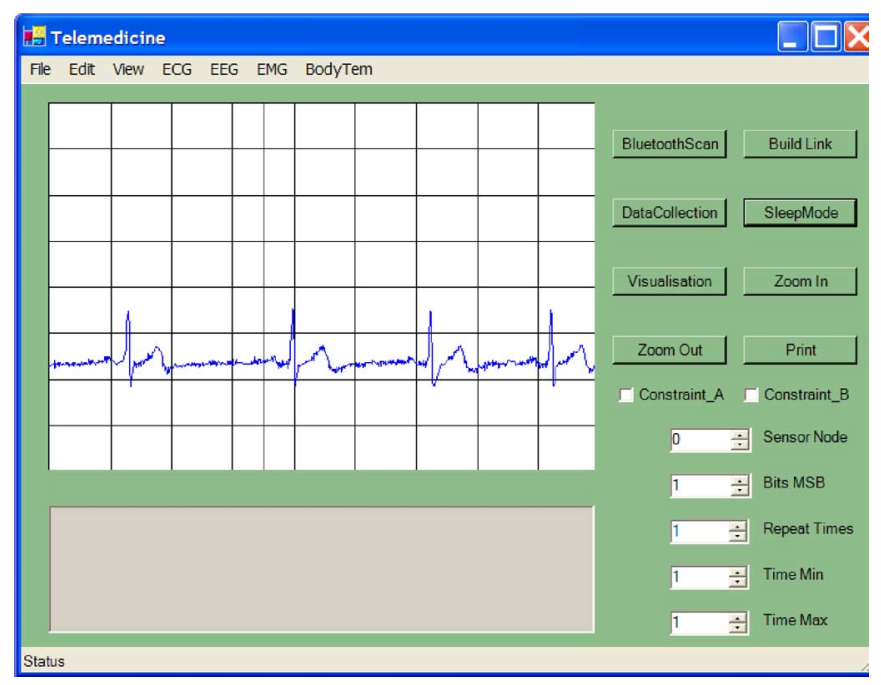

Fig. 14. Look and feel of the user interface.

at home, each sensor node collects a kind of physiological signal. The wireless medical sensor nodes attached to the patient send data to the gateway module, forming a short-range Bluetooth wireless network. The coordinator node is connected to the home computer through a USB interface. The home computer, already connected to the Internet, can establish a secure channel to the medical server and send periodic updates for the patient's medical record and for medical personnel replying to the diagnostic advices. In this configuration, the coordinator node, a part of gateway module, always acts as a master, and the maximum number of the medical sensor nodes that can be active for physiological signals measuring is 7. Because this initial evaluation aims only for verifying practicality of the adopted approach and the overall system architecture, it used only two sensor nodes: each having only ECG sensor. Two persons' ECG signals were monitored.

For the deployment in a hospital, some coordinator nodes are distributed in the wards of a hospital. These coordinator nodes are connected to the main medical server through a computer in the hospital. The coordinator node always acts as a master in each piconet. Each patient takes a comprehensive medical sensor node that integrates the functions of collecting several physiological signals. The medical sensor node always acts as a slave in a piconet. In this way, the physiological signals can be monitored continuously by medical personnel in real time through the deployment of these unobtrusive medical sensors, thus avoiding tethering the patients to the conventional medical equipment. With the same aim as the aforementioned evaluation, the system configuration of this initial evaluation used six coordinator nodes; each piconet has two ECG sensor nodes. In total, 12 persons' ECG signals were monitored.

The initially built system has been tested (see Fig. 13). The look and feel of the user interface at home computer of a patient is shown in Fig. 14.

\section{CONCLUSION}

A Bluetooth-based wireless sensor network has been developed for continuously monitoring the physiological signals of a patient. This new technology has potential for offering a wide range of benefits to patients, medical personnel, and society through continuous monitoring feature, early detection of abnormalities with high reliability and security, and potential knowledge discovery through data mining of all collected medical data. There are many advantages of adopting wireless remote healthcare at home or in hospitals to replace the face-to-face communication between a patient and a physician. First, medical personnel can get the patients' physiological information in time, and then, give real-time diagnostic advices that are important to patients' recovery. Second, patients can measure their physiological signals, and then, send to the hospital remotely without the need of going to hospital in person. Third, patients can move around freely while carrying wireless medical devices. Furthermore, with the help of this system, medical personnel can take care of a few patients simultaneously, and thus, the personnel expenses can be reduced.

Our research and evaluation clearly demonstrates that currently available systems for monitoring physiological signals suffer from technical weakness and limitations, resulting in under-exploitation of potentially lifesaving data. Our innovative approach creates a system infrastructure that both decreases the cost involving with monitoring patients and increases the efficient exploitation of physiological data. As a result of this system, patients can be benefited by high-quality medical care in their own home, thus avoiding the distress and disruption caused by a lengthy impatient stay in hospital.

\section{REFERENCES}

[1] European Observatory on Health Systems and Policies. (2007, Dec.). Health Care Outside Hospital Accessing Generalist and Specialist Care in Eight Countries [Online]. Available: http://www.euro.who.int/ Document/E89259.pdf

[2] Department of Health, National Health Service (NHS). (2007, Dec.) Our Health, Our Care, Our Say: A New Direction for Community Service [Online]. Available: http://www.ehiprimarycare.com/tc_domains Bin/DocumentLibrary0282/Community_care_white_paper_30-1-06.pdf

[3] T. Martin, E. Jovanov, and D. Raskovic, "Issues in wearable computing for medical monitoring applications: A case study of a wearable ECG monitoring device," presented at the Int. Symp. Wearable Comput. 2000 (ISWC 2000), Atlanta, GA, Oct. 
[4] C. Otto, A. Milenkovic, C. Sanders, and E. Jovanov, "System architecture of a wireless body area network for ubiquitous health monitoring," $J$. Mobile Multimedia, vol. 1, no. 4, pp. 307-326, 2006.

[5] A. Milenkovic, C. Otto, and E. Jovanov, "Wireless sensor network for personal health monitoring: Issues and an implementation," J. Comput. Commun., vol. 29, no. 13/14, pp. 2521-2533, 2006.

[6] M. F. A. Rasid and B. Woodward, "Bluetooth telemedicine processor for multi-channel biomedical signal transmission via mobile cellular networks," IEEE Trans. Inf. Technol. Biomed., vol. 9, no. 1, pp. 35-43, Mar. 2005.

[7] V. Shnayder, B. Chen, K. Lorincz, T. R. F. Fulford-Jones, and M. Welsh, "Sensor networks for medical care," Div. Eng. Appl. Sci., Harvard Univ., Cambridge, MA, Tech. Rep. TR-08-05, 2005.

[8] D. Blasi, V. Cacace, L. Casone, M. Rizzello, S. Rotolo, and L. Bononi, "Ad hoc wireless sensor networking: Challenges and issues," ST J. Res., vol. 4, no. 1, pp. 19-32, 2006.

[9] R. S. H. Istepanian, L. J. Hadjileontiadis, and S. M. Panas, "ECG data compression using wavelets and higher order statistics methods," IEEE Trans. Inf. Technol. Biomed., vol. 5, no. 2, pp. 108-115, Jun. 2001.

[10] R. S. H. Istepanian and A. A. Petrosian, "Optimal zonal wavelet-based ECG data compression for a mobile telecardiology system," IEEE Trans. Inf. Technol. Biomed., vol. 4, no. 3, pp. 200-211, Sep. 2000.

[11] S. Zhou, G. Guillemette, R. Antinoro, and F. Fulton, "New approaches in Philips ECG database management system design," Comput. Cardiol., vol. 30, pp. 267-270, 2003.

[12] H. Jumaa, J. Fayn, and P. Rubel, "XML based mediation for automating the storage of SCP-ECG data into relational databases," Comput. Cardiol. vol. 35, pp. 445-448, 2008.

[13] H. S. Liu, T. Zhang, and F. S. Yang, "A multistage, multi-method approach for automatic detection and classification of epileptiform EEG," IEEE Trans. Biomed. Eng., vol. 49, no. 12, pp. 1557-1566, Dec. 2002.

[14] N. Acir, "A modified hybrid neural network for pattern recognition and its application to SSW complex in EEG," Neural Comput. Appl., vol. 15, no. 1, pp. 49-54, 2005.

[15] P. Bonato, P. J. Mork, D. M. Sherrill, and R. H. Westgaard, "Data mining of motor patterns recorded with wearable technology," IEEE Eng. Med. Biol. Mag., vol. 22, no. 3, pp. 110-119, May/Jun. 2003.

[16] J. J. Segura-Juárez, D. Cuesta-Frau, L. Samblas-Pena, and M. Aboy, “A microcontroller-based portable electrocardiograph recorder," IEEE Trans. Biomed. Eng., vol. 51, no. 9, pp. 1686-1690, Sep. 2004.

[17] D. Ganesan, A. Cerpa, W. Ye, Y. Yu, J. Zhao, and D. Estrin, "Networking issues in wireless sensor networks," J. Parallel Distrib. Comput., vol. 64, no. 7, pp. 799-814, 2004.

[18] E. Spinelli, R. Pallas-Areny, and M. Mayosky, "AC-coupled front-end for bio-potential measurements," IEEE Trans. Biomed. Eng., vol. 50, no. 3, pp. 391-395, Mar. 2003.

[19] D. Malan, T. Fulford-Jones, M. Welsh, and S. Moulton, "CodeBlue: An ad hoc sensor network infrastructure for emergency medical care," presented at the MobiSys 2004 Workshop Appl. Mobile Embedded Syst. (WAMES 2004), Boston, MA.

[20] C. Park, P. H. Chou, Y. Bai, R. Matthews, and A. Hibbs, "An ultrawearable, wireless, low power ECG monitoring system," in Proc. IEEE Biomed. Circuits Syst. Conf. (BioCAS 2006), Dec., pp. 241-244.

[21] C. C. Chiang, W. C. Tzeng, H. C. Cheng, C. T. Lin, Y. C. Yang, S F. Liang, and S. B. Lim, "Construction and application of an electronic ECG management system," J. Inf. Technol. Appl., vol. 2, no. 3, pp. 135140,2007

[22] BTnodes-A Distributed Environment for Prototyping Ad Hoc Networks. (2007, Dec.). [Online]. Available: http://www.btnode.ethz.ch/

[23] J. Beutel, M. Dyer, M. Hinz, L. Meier, and M. Ringwald, "Next-generation prototyping of sensor networks," in Proc. 2nd ACM Int. Conf. Embedded Netw. Sens. Syst. (SenSys 2004), Nov., pp. 291-292.

[24] J. Beutel, O. Kasten, F. Mattern, K. Romer, F. Siegemund, and L. Thiele, "Prototyping wireless sensor network applications with BTnodes," in Proc. 1st Eur. Workshop Sens. Netw. (EWSN 2004) Jan., pp. 323338.

[25] J. Beutel, "Fast-prototyping using the BTnode platform," in Proc. Conf. Des., Autom. Test Eur. (DATE 2006), Mar., pp. 977-982.

[26] J. Eliasson, P. Lindgren, and J. Delsing, "A Bluetooth-based sensor node for low-power ad hoc networks," J. Comput., vol. 3, no. 5, pp. 1-10, 2008.

[27] Chipcon CC1000 Datasheet. (2008, Dec.). [Online]. Available: http:// www.chipcon.com/

[28] ZV4002 Datasheet, Zeevo Corporation, Santa Clara, CA, 2004.

[29] Real Time Operating System. (2008, Dec.). [Online]. Available: http://www.ethernut.de/en/software.html
[30] J. Polastre, R. Szewczyk, and D. Culler, "Telos: Enabling ultra-low power wireless research," in Proc. 4th Int. Symp. Inf. Process. Sens. Netw. (IPSN 2005), Apr., pp. 364-369.

[31] Telos (Rev B) Datasheet, Moteiv Corporation, Redwood City, CA, 2004.

[32] Chipcon CC2420 Datasheet. (2008, Dec.). [Online]. Available: http:// www.chipcon.com/

[33] (2008, Dec.). MSP430F1611 Datasheet [Online]. Available: http:// www.ti.com/

[34] P. Levis, S. Madden, J. Polastre, R. Szewczyk, K. Whitehouse, A. Woo, D. Gay, J. Hill, M. Welsh, E. Brewer, and D. Culler, "TinyOS: An operating system for wireless sensor networks," in Ambient Intelligence. New York: Springer-Verlag, 2004.

[35] R. Kling, R. Adler, J. Huang, V. Hummel, and L. Nachman, "Intel mote: Using Bluetooth in sensor networks," in Proc. 2nd Int. Conf. Embedded Netw. Sens. Syst. (SenSys 2004), Nov., pp. 318-318.

[36] L. L. Chan, J. Z. Zhang, M. Narayanan, B. G. Celler, and N. H. Lovell, "A health monitoring and evaluation system for assessing care needs of residents in aged-care facilities," in Proc. 6th IASTED Int. Conf. Biomed. Eng. (BioMed 2008), Feb., pp. 145-189.

[37] I. Gondal, M. Iqbal, M. Woods, and S. Sehgal, "Integrated sensing and diagnosis-The next step in real time patient health care," in Proc. 6th IEEE/ACIS Int. Conf. Comput. Inf. Sci. (ICIS 2007), Jul., pp. 581-586.

[38] F. Spadini, F. Vergari, L. Nachman, L. Lamberti, and T. S. Cinotti, "A wireless and context-aware ECG monitor: An iMote2 based portable system," Comput. Cardiol., vol. 35, pp. 997-1000, 2008.

[39] W. Featherstone and D. Molkdar, "Capacity benefits of GPRS coding schemes CS-3 and CS-4," 3G Mobile Commun. Technol., vol. 489, pp. 287-291, May 2002.

[40] GPRS White Paper, Cisco Systems, San Jose, CA, 2000.

[41] (2007, Dec.). Bluetooth specification [Online]. Available: http://www. bluetooth.com/

[42] (2007, Dec.). Specification of the Bluetooth System: Core, Part B Base-Band Specification (Version 1.1) [Online]. Available: http://www. bluetooth.com/

[43] P. R. Suri and S. Rani, "Bluetooth authentication and personal identification number estimation by attacker," Inf. Technol. J., vol. 6, no. 6, pp. 938-940, 2007.

[44] CP3SP33 Datasheet, National Semiconductor Corp., Santa Clara, CA, 2007.

[45] LMX5252 Datasheet, National Semiconductor Corp., Santa Clara, CA, 2003.

[46] (2007, Dec.). INA321 Datasheet [Online]. Available: http://www.ti.com/

[47] J. Labrosse, MicroC/OS-II: The Real-Time Kernel, 2nd ed. Gilroy, CA: CMP Books.

[48] Bluetooth Software User's Manual, National Semiconductor Corp's Documents, Santa Clara, CA, 2007.

[49] M. Cortiana, A. Labombarda, and L. Vanzago, "Cupola sensing: An indoor climate monitoring test case," ST J. Res., vol. 4, no. 1, pp. 52-64, 2006.

Ying Zhang received the B.Eng. degree in communication engineering from Xi' an Jiaotong University, Xi' an, China, in 1990, and the Ph.D. degree in information systems from the University of Salford, Salford, U.K., in 2005.

From 1990 to 1999, he was with Xi' an Microelectronics Technology Institute, China Aerospace Corporation, China. Since coming to U.K. in 1999 as a Visiting Scholar sponsored by the China Scholarship Council, he has ever held research position at the University of Manchester, the University of Glasgow, the University of Hertfordshire, and the University of Cambridge. He has authored or coauthored more than 30 papers published in books and journals, and presented at conferences. His research interests include human-computer interaction, multimodal virtual environment technology, perceptive user interface, interactive visualization, wireless sensor network, and ubiquitous computing applications.

Hannan Xiao received the B.Eng. degree in 1992 and M.Eng. degree in 1995, both in electronics and information system engineering from the Huazhong University of Science and Technology, Hubei, China, and the Ph.D. degree in electrical and computer engineering from the National University of Singapore, Singapore, in 2003.

She was a hands-on network engineer at the Central China Regional Network Center of China Education and Research NETwork (CERNET) from 1995 to 1998, and a Research Fellow with the National University of Singapore from 2002 to 2003. Since October 2003, She has been with the School of Computer Science, University of Hertfordshire, Hatfield, U.K. Her research interests are in the areas of wireless networks and mobile computing, network security, network congestion control, quality of service, admission control, ad hoc networks, and sensor networks. 